\title{
A search for changing-look AGN in the Grossan catalog
}

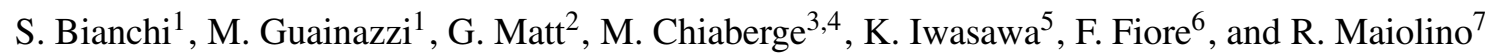 \\ 1 XMM-Newton Science Operations Center, European Space Astronomy Center, ESA, Apartado 50727, 28080 Madrid, Spain \\ e-mail: Stefano.Bianchi@sciops.esa.int \\ 2 Dipartimento di Fisica, Università degli Studi Roma Tre, Via della Vasca Navale 84, 00146, Roma, Italy \\ 3 Space Telescope Science Institute, 3700 San Martin Drive, Baltimore, MD 21218, USA \\ 4 INAF - Istituto di Radioastronomia, Via P. Gobetti 101, 40129 Bologna, Italy \\ 5 Institute of Astronomy, Madingley Road, Cambridge, CB3 OHA, UK \\ ${ }^{6}$ INAF - Osservatorio Astronomico di Roma, via di Frascati 33, 00040 Monteporzio, Italy \\ 7 INAF - Osservatorio Astrofisico di Arcetri, Largo Fermi 5, 50125 Firenze, Italy
}

Received 9 May 2005 / Accepted 13 July 2005

\section{ABSTRACT}

We observed with XMM-Newton 4 objects selected from the Grossan (1992, Ph.D. Thesis) catalog, with the aim to search for new "changinglook" AGN. The sample includes all the sources which showed in subsequent observations a flux much lower than the one measured with HEAO A-1: NGC 7674, NGC 4968, IRAS 13218+0552 and NGC 1667. None of the sources was caught in a high flux state during the XMMNewton observations, whose analysis reveal they are all likely Compton-thick objects. We suggest that, for all the sources, potential problems with the HEAO A-1 source identification and flux measurement prevent us from being certain that the HEAO A-1 data represent a putative "high" state for these objects. Nonetheless, based on the high flux state and Compton-thin spectrum of its GINGA observation, NGC 7674 represents probably the sixth known case of a "changing-look" Seyfert 2 galaxy. From the X-ray variability pattern, we can estimate a likely lower limit of a few parsec to the distance of the inner walls of the torus in this object. Remarkably, IRAS 13218+0552 was not detected by XMM-Newton, despite being currently classified as a Seyfert 1 with a large [OIII] flux. However, the original classification was likely to be affected by an extreme velocity outflow component in the emission lines. The object likely harbors an highly obscured AGN and should be re-classified as a type 2 source.

Key words. galaxies: Seyfert - X-rays: galaxies - X-rays: individual: NGC 7674 - X-rays: individual: IRAS 13218+0552

\section{Introduction}

Recently, a few Seyfert 2 galaxies were discovered to make transitions, on time scales of a few years, from a Comptonthin appearance (when the nuclear radiation is absorbed by material with a line-of-sight column density less than $\sigma_{T}^{-1}=$ $\left.1.5 \times 10^{24} \mathrm{~cm}^{-2}\right)$ to a reflection-dominated spectrum, and/or vice versa (Guainazzi et al. 2002; Guainazzi 2002; Matt et al. 2003). A reflection-dominated spectrum is recognized by a hard continuum and a prominent (Equivalent Width, EW, $\sim 1 \mathrm{keV}$ ) iron line, and it is commonly assumed to be a signature of Compton-thick absorption (Matt 2000, 2002a). In this hypothesis, the reflection would be due to circumnuclear material, including the part of the absorbing matter which is visible to both the nucleus and the observer, such as the far side of the inner wall of the torus envisaged in Unification Models (Antonucci 1993).

Two possibilities exist for the observed transitions: either a change in the line-of-sight absorbing column, or a "switchingoff" of the nucleus, leaving reflection from distant matter as an echo of past activity. While the first hypothesis cannot in most cases be ruled out (and, indeed, seems to be the best explanation in the case of NGC 1365: Risaliti et al. 2005), the second appears more likely (see discussion in Matt et al. 2003) and in at least one case, namely NGC 2992 (Gilli et al. 2000), it is the only tenable. These sources would then be the Seyfert 2 analogs of the Narrow Line Seyfert 1 NGC 4051 (Guainazzi et al. 1998; Uttley et al. 1999). In the following, we will refer to all sources (whether Seyfert 1s or 2s) with the term "changing look", if they temporarily appear to be reflection-dominated.

These objects have been serendipitously discovered; the very nature of this transition makes a systematic study of their properties difficult. In particular, how frequently sources undergo this sort of transition? Moreover, how many Comptonthick Seyfert 2 are really heavily absorbed, and not simply switched-off? It is quite difficult to estimate the fraction of these transitions, due to the lack of a complete and unbiased sample of homogeneously defined Seyfert galaxies with sufficient X-ray temporal and spectroscopic coverage. A first step in this direction was done by Guainazzi et al. (2005a) who reported a typical occurrence rate of a transition every 50 years, on the basis of a sample of 11 optically-selected Seyfert 2 galaxies, whose $A S C A$ and/or BeppoSAX observations 
Table 1. The sources included in our sample and the log of the XMM-Newton observations.

\begin{tabular}{ccccccccc}
\hline \hline Name & LASS Name & $z$ & Type (NED) & {$\left[_{\text {OIII }}\right]^{a}$} & IR $^{b}$ & XMM Obs. Date & ObsID & Exp. (ks) \\
\hline NGC 7674 & 1H2320+084 & 0.029 & Sey 2 & 1.9 & 4.7 & $2004-06-02$ & 0200660101 & 9 \\
NGC 4968 & $1 \mathrm{H} 1308-237$ & 0.01 & Sey 2 & 11.1 & 2.7 & $2001-01-05$ & 0002940101 & 4 \\
& & & & & & $2004-07-05$ & 0200660201 & 10 \\
IRAS 13218+0552 & 1H1320+066 & 0.21 & Sey 1 & 0.73 & 1.1 & $2004-07-11$ & 0200660301 & 10 \\
NGC 1667 & 1H0445-060 & 0.015 & Sey 2 & 2.0 & 3.8 & $2004-09-20$ & 0200660401 & 8 \\
\hline
\end{tabular}

${ }^{a}$ [OIII] fluxes in units of $10^{-12} \mathrm{erg} \mathrm{cm}^{-2} \mathrm{~s}^{-1}$, corrected for extinction adopting the prescription given by Bassani et al. (1999). All data were taken from Polletta et al. (1996), except for IRAS 13218+0552 (Kim et al. 1998). ${ }^{b}$ Far-infrared fluxes in units of $10^{-10} \mathrm{erg} \mathrm{cm}^{-2} \mathrm{~s}^{-1}$, defined as $F_{\mathrm{IR}}=F_{25 \mu \mathrm{m}} \times v_{25 \mu \mathrm{m}}+F_{60 \mu \mathrm{m}} \times v_{60 \mu \mathrm{m}}$. All data were taken from Polletta et al. (1996), except for IRAS 13218+0552 (Kim \& Sanders 1998).

suggested Compton-thick obscuration. In this paper, we select a mini-sample of other four "changing-look" candidates with long-term X-ray coverage.

\section{Observations}

\subsection{The sample}

Our sample is extracted from the Grossan (1992) catalog, based on the catalog of X-ray sources from the LASS (Large Area Sky Survey, or HEAO A-1) instrument aboard the HEAO-1 satellite, which included the first scan of the full sky from August 1977 to February 1978 (Wood et al. 1984). The catalog took advantage of the overlapping diamond-shaped error regions of the MC instrument (Modulation Collimator, or A-3 experiment: Gursky et al. 1978), which also flew aboard the HEAO-1 satellite, to identify the 96 AGN that make up the socalled LMA (LASS/MC identified AGN) sample, down to a limiting 2-10 keV flux of about $1.8 \times 10^{-11} \mathrm{erg} \mathrm{cm}^{-2} \mathrm{~s}^{-1}$.

Our sample selection criteria aimed at finding good candidates for "changing-look" sources, to be observed with XMM-Newton. The selection was made by taking all the sources in the Grossan catalog which have subsequently been observed in X-rays to be reflection-dominated, or too faint to allow for a detailed spectral analysis, with a flux at least a factor 50 fainter than observed by HEAO A-1. If the optical identifications are correct, these sources could therefore be "changinglook" sources.

These selection criteria resulted in a mini-sample of four targets: NGC 7674, NGC 4968, IRAS 13218+0552 and NGC 1667. We note here that none of these sources belongs to the Piccinotti et al. (1982) HEAO A-2 sample, because they have all 2-10 keV fluxes below its limit.

\subsection{Data reduction}

Table 1 shows the log of all the XMM-Newton observations analysed in this paper. All observations were performed with the EPIC CCD cameras, the pn (Strüder et al. 2001) and the two MOS (Turner et al. 2001), but with different combinations of subframes and filters. Data were reduced with SAS 6.1.0 (Gabriel et al. 2004) and screening for intervals of flaring particle background was done consistently with the choice of extraction radii, in an iterative process based on the procedure to maximize the signal-to-noise ratio described by
Piconcelli et al. (2004). No source suffers from pileup problems, so pn spectra were extracted with pattern 0 to 4 and MOS spectra with patterns 0 to 12 . When the two MOS observations were performed with the same subframe, their spectra were summed. All spectra were binned in order to oversample the instrumental resolution by at least a factor of 3 and to have no less than 25 counts in each background-subtracted spectral channel. The latter requirement allows us to use the $\chi^{2}$ statistics. On the other hand, "local fits" were also performed in the 5.25-7.25 keV energy range with the unbinned spectra, using the Cash (1976) statistics, in order to better assess the nature or the presence of the iron lines, like in the cases of NGC 1667 and NGC 7674. We refer the reader to Guainazzi et al. (2005b) for details on this kind of analysis. However, all the $E W$ s reported in the paper refer to the global fits on the binned spectra and with respect to the best fit model.

For two sources, namely NGC 7674 and IRAS $13218+0552$, we also analysed previous BeppoSAX observations, in the latter case for the first time, to better compare them to the XMM-Newton results. Event files and spectra were retrieved from the ASDC Multi-Mission Interactive Archive ${ }^{1}$. MECS spectra for NGC 7674 were also re-extracted from circular regions with different radii, using XSELECT. As for the ASCA observations of NGC 4968 and NGC 1667, since they are characterised by detections at the limit of the instrument and different fluxes are reported in literature, we re-analyzed archival data, starting from linearized event lists extracted from the HEASARC archive ${ }^{2}$.

\subsubsection{A note on GINGA observations}

Since the GINGA observation of NGC 7674 will be of fundamental importance to understand the nature of this source, we need to consider with some detail the reliability of GINGA fluxes of faint objects. The brightness of the X-ray background (XRB) is known to fluctuate on the sky. With the beam of the GINGA LAC $\left(F W H M=1^{\circ} \times 2^{\circ}\right), 3 \sigma$ fluctuation of the XRB was estimated to be $\sim 2 \mathrm{ct} \mathrm{s}^{-1}$ in the $2-10 \mathrm{keV}$ band (Hayashida et al. 1989; Butcher et al. 1997). Therefore, in the absence of a reliable estimate of the local XRB brightness level, the cosmic

\footnotetext{
1 http://www .asdc.asi.it/

2 http://heasarc.gsfc.nasa.gov/db-perl/W3Browse/ w3browse.pl
} 
variance of the XRB limits the source detection with a pointed GINGA observation.

For some sources, scanning observations were made just before or after pointing at the target object. This will secure the source identification at least in the direction of the scan path within 0.2 degree or less, and reduce the uncertainty due to the cosmic variance of the XRB brightness.

There is a scanning observation for NGC 7674, in which a significant excess was detected at the position of the galaxy, with a count rate of $3.3 \mathrm{ct} \mathrm{s}^{-1}$ (Awaki 1991). The XRB level was estimated based on the scanning data in Awaki (1991). On the other hand, Smith \& Done (1996) did not consider NGC 7674 to be detected because the source count rate they estimated was smaller than the cosmic variance $\left(1.8 \mathrm{ct} \mathrm{s}^{-1}\right)$. Awaki (1991) and Smith \& Done (1996) used different methods in estimating the detector background. This might introduce some difference in their measure of the source flux. However, we consider the source detection reported in Awaki (1991) to be reliable, as it was based on the scanning observation, which is free from the uncertainty due to cosmic variance in the XRB.

On the other hand, no scanning observation was carried out for NGC 1667 while the local XRB was estimated only based on the data taken at the nearby off-source sky. The source count rate for NGC 1667 reported in Awaki \& Koyama (1993) is $0.5 \mathrm{ct} \mathrm{s}^{-1}$, which is smaller than the cosmic variance of the $\mathrm{XRB}$. Because of the lack of a scanning observation, this small excess recorded for NGC 1667 cannot be considered as a reliable detection and we take this value as an upper limit. An earlier detection reported in Polletta et al. (1996) came from a detection of a source during a slewing operation of the satellite. The detection is only in the soft band $(2-4 \mathrm{keV})$. An inspection of the ROSAT All-Sky Survey (RASS) image finds brighter soft $\mathrm{X}$-ray sources: one at 15 arcmin to the $\mathrm{E}$ and two in 75 arcmin to the $\mathrm{N}$ and SE, and they are likely to be confused with NGC 1667.

All spectra were analyzed with XSPEC 11.3.1. In the following, errors correspond to the $90 \%$ confidence level for one interesting parameter $\left(\Delta \chi^{2}=2.71\right)$, where not otherwise stated. The cosmological parameters used throughout this paper are $H_{0}=70 \mathrm{~km} \mathrm{~s}^{-1} \mathrm{Mpc}^{-1}, \Lambda_{0}=0.73$ and $q_{0}=0$.

\section{Analysis}

\subsection{NGC 7674}

NGC 7674 is a Seyfert 2 galaxy with broad $\mathrm{H} \alpha$ and $\mathrm{H} \beta$ components in polarized light (Miller \& Goodrich 1990; Young et al. 1996). After its LASS 2-10 keV flux of $2.4 \times 10^{-11} \mathrm{erg} \mathrm{cm}^{-2} \mathrm{~s}^{-1}$, Awaki et al. (1991) reported a flux of $8 \times 10^{-12} \mathrm{erg} \mathrm{cm}^{-2} \mathrm{~s}^{-1}$ with GINGA, with a spectral shape characterized by an absorbed powerlaw (but the derived column density was unconstrained) and an upper limit of $80 \mathrm{eV}$ to the iron line $E W$. Subsequently, a ROSAT PSPC spectrum derived a $0.5-2 \mathrm{keV}$ flux of $2 \times 10^{-13} \mathrm{erg} \mathrm{cm}^{-2} \mathrm{~s}^{-1}$ (Levenson et al. 2001). The source was then observed by BeppoSAX on November 1996, and found clearly reflection-dominated, with a flux of $5 \times 10^{-13} \mathrm{erg} \mathrm{cm}^{-2} \mathrm{~s}^{-1}$ (Malaguti et al. 1998). If the source was absorbed, instead of switched-off as suggested by

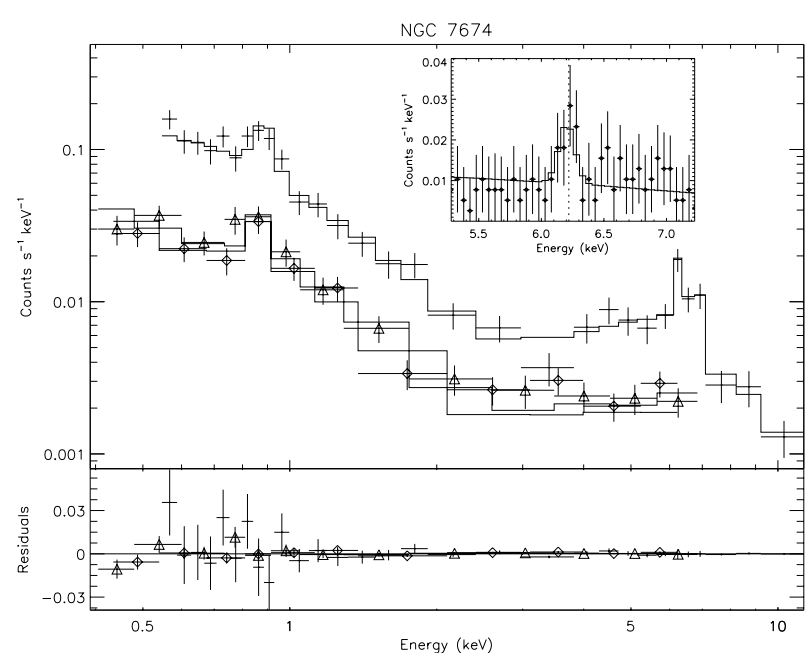

Fig. 1. NGC 7674: XMM-Newton spectra (pn: crosses, MOS1: diamonds, MOS2: triangles) and best fit model. In the inset: "local fit" on the background-subtracted, unbinned pn spectrum around the restframe iron line energy, marked with a broken line (see text for details).

the HEAO A-1 and GINGA measurements, the lack of strong excess emission in the PDS instrument permits to put a lower limit to the column density of the absorber of several times $10^{24} \mathrm{~cm}^{-2}$

XMM-Newton confirmed the spectral shape observed by BeppoSAX, which we modeled with a bare Compton reflection component (model PEXRAV in XSPEC: Magdziarz \& Zdziarski 1995), a neutral iron $\mathrm{K} \alpha$ line and a steep power law for the soft excess, with photon index $\Gamma_{\mathrm{s}}$. Two further emission lines are required by the data, at $0.91_{-0.03}^{+0.02} \mathrm{keV}(\mathrm{Ne}$ IX K $\alpha$ ) and at $6.97_{-0.05}^{+0.26} \mathrm{keV}$, at the $99.9 \%$ and $98 \%$ confidence level, respectively, according to F-test. The latter confirms the complex iron line profile observed with BeppoSAX, but is not unambiguously present in the "local fit" (see inset in Fig. 1): a likely explanation is that the feature is not dominated by a single emission line, but is instead a blend of lines, such as the Fe XXVI $\mathrm{K} \alpha$ and the neutral $\mathrm{Fe} \mathrm{K} \beta$.

The only problem with a Compton-reflection dominated scenario is the $E W$ of the neutral iron line $(\simeq 400 \mathrm{eV}$ with respect to the reflection component only), which is much lower than the expected one, i.e. $\gtrsim 1 \mathrm{keV}$ (see e.g. Matt et al. 1996). It is likely that the measure of the flux of the iron line in NGC 7674 is affected by the above-mentioned prominent feature at higher energies, considering that the EPIC spectra do not have high statistics. In any case, $E W$ as low as $\simeq 600 \mathrm{eV}$ have been measured in Compton-thick sources and may be due to iron underabundance and/or a small inclination angle of the torus (see e.g. Mrk 3: Bianchi et al. 2005).

The XMM-Newton spectrum is plotted in Fig. 1, while Table 2 summarizes the best fit parameters compared to our re-analysis of the BeppoSAX data. No significant variability is found in any of the spectral parameters. Moreover, we find a somewhat larger BeppoSAX 2-10 keV flux with respect to the one reported by Malaguti et al. (1998) (but they do not quote any error), in better agreement with the $7 \times 10^{-13} \mathrm{erg} \mathrm{cm}^{-2} \mathrm{~s}^{-1}$ measured with XMM-Newton. We note that the BeppoSAX flux should not be contaminated by nearby sources, as the 

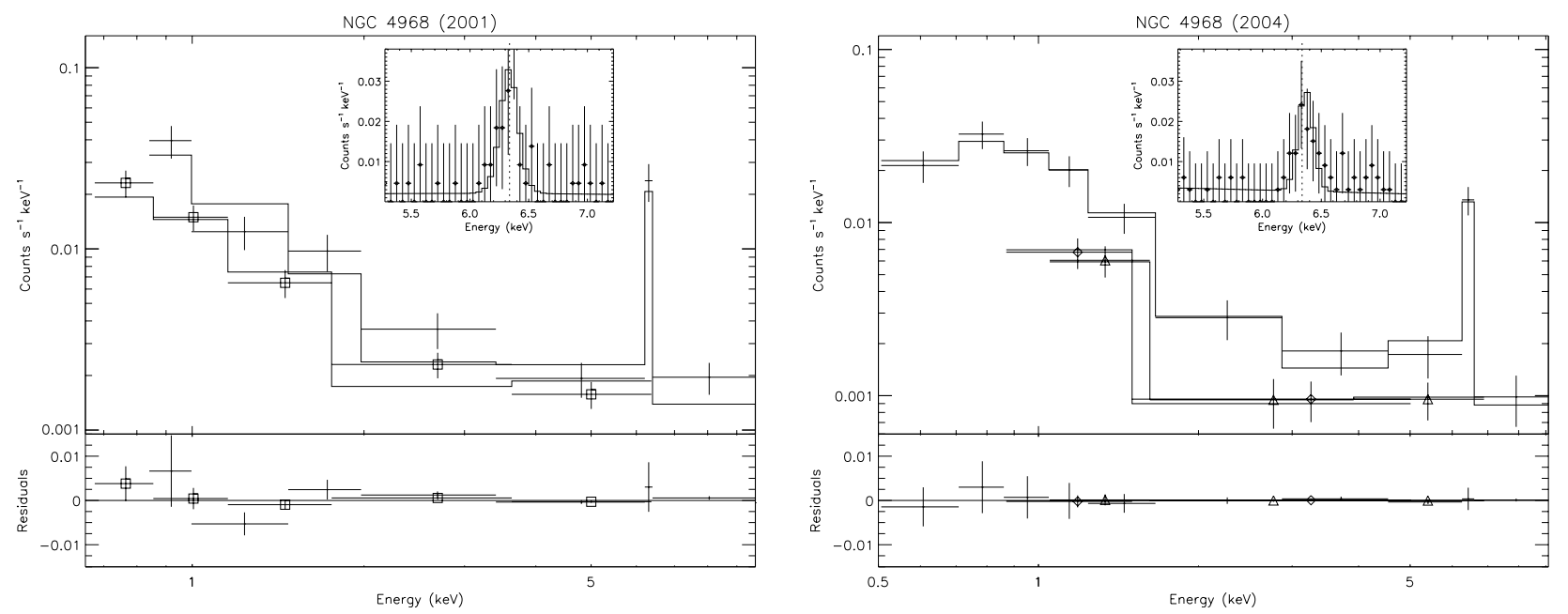

Fig. 2. NGC 4968: data ( $p n$ : crosses, MOS1: diamonds, MOS2: triangles, combined MOS: squares) and best fit model for the first (left) and the second (right) XMM-Newton observation. In the insets: "local fits" on the background-subtracted, unbinned pn spectra around the rest-frame iron line energy, marked with a broken line (see text for details).

Table 2. NGC 7674: best fit parameters for the observations analyzed in this paper (see text for details).

\begin{tabular}{c|cc}
\hline \hline & SAX & XMM \\
& $(1996)$ & $(2004)$ \\
\hline$F_{2-10}\left(10^{-12} \mathrm{erg} \mathrm{cm}^{-2} \mathrm{~s}^{-1}\right)$ & $0.75 \pm 0.06$ & $0.70 \pm 0.07$ \\
$\Gamma$ & $2.05_{-0.18}^{+0.19}$ & $1.7_{-0.2}^{+0.3}$ \\
$\Gamma_{\mathrm{s}}$ & $4.6_{-0.8}^{+1.2}$ & $3.3 \pm 0.2$ \\
$E W_{6.4}(\mathrm{keV})^{630_{-230}^{+200}}$ & $370_{-170}^{+160}$ \\
$F_{6.4}\left(10^{-6} \mathrm{ph} \mathrm{s}^{-1}\right)$ & $7.7_{-2.8}^{+2.4}$ & $4.2_{-1.9}^{+1.8}$ \\
$E W_{6.97}(\mathrm{keV})^{-1.20}$ & $340_{-230}^{+200}$ & $480_{-300}^{+220}$ \\
$F_{6.97}\left(10^{-6} \mathrm{ph} \mathrm{s}^{-1}\right)$ & $3.7_{-2.4}^{+2.1}$ & $4.8_{-3.0}^{+2.2}$ \\
$\chi^{2} /$ d.o.f. & $63 / 59$ & $45 / 44$ \\
\hline
\end{tabular}

selected extraction radius of 2 arcsec is free of other bright sources (see Table 6). No short-term variability is found in the XMM-Newton lightcurves.

As a final comment, we would like to point out that it is quite difficult to find a physical origin for the very steep powerlaw needed to fit the soft X-ray spectrum of NGC 7674. This is a common problem in the analysis of Compton-thick Seyfert galaxies but, as already suggested by Iwasawa et al. (2002) and Guainazzi et al. (2004), it may be the result of a blending of strong emission lines which mimic a continuum component in low resolution spectra. Indeed, this interpretation turns out to be true in the few objects were high resolution spectra are available, as in NGC 1068 (Kinkhabwala et al. 2002; Brinkman et al. 2002), Circinus (Sambruna et al. 2001) and Mrk 3 (Bianchi et al. 2005; Sako et al. 2000). This seems also to be the case for NGC 7674. A fit of equivalent statistical quality $\left(\chi^{2}=39 / 37\right.$ d.o.f. $)$ is achieved by modelling the soft spectrum with a powerlaw with the same photon index of the primary continuum (now $\Gamma \simeq 1.85$ ), plus a number of emission lines, mostly from $\mathrm{H}$ - and $\mathrm{H}$-like $\mathrm{O}, \mathrm{Ne}, \mathrm{Mg}$ and $\mathrm{Si}$. Therefore, an interpretation of the soft X-ray spectrum in terms of emission from a gas photoionized by the nuclear continuum is the most
Table 3. NGC 4968: best fit parameters for the observations analyzed in this paper (see text for details).

\begin{tabular}{c|cc}
\hline \hline & XMM1 & XMM2 \\
& $(2001)$ & $(2004)$ \\
\hline$F_{2-10}\left(10^{-12} \mathrm{erg} \mathrm{cm}^{-2} \mathrm{~s}^{-1}\right)$ & $0.27 \pm 0.08$ & $0.23 \pm 0.08$ \\
$\Gamma$ & $1.7^{*}$ & $1.7^{*}$ \\
$\Gamma_{\mathrm{s}}$ & $3.4_{-0.6}^{+0.7}$ & $2.7 \pm 0.3$ \\
$E W_{6.4}(\mathrm{eV})$ & $1900 \pm 900$ & $3200 \pm 1100$ \\
$F_{6.4}\left(10^{-6} \mathrm{ph} \mathrm{s}^{-1}\right)$ & $7.1_{-3.4}^{+3.1}$ & $7.3_{-2.5}^{+2.4}$ \\
$\chi^{2} /$ d.o.f. & $17 / 10$ & $9 / 10$ \\
\hline
\end{tabular}

${ }^{*}$ Fixed.

likely one also in this object, even if high resolution spectra would be required to finally clarify this issue.

\subsection{NGC 4968}

NGC 4968 is a Seyfert 2 galaxy which was not detected by GINGA (Awaki 1991) or in the RASS, with an upper limit on the PSPC count rate of 0.03 , corresponding roughly to a $0.5-2 \mathrm{keV}$ flux of $10^{-13} \mathrm{erg} \mathrm{cm}^{-2} \mathrm{~s}^{-1}$, assuming a $\Gamma=1.7$ powerlaw spectrum (Rush et al. 1996). A $2-10 \mathrm{keV}$ flux of a few $10^{-13} \mathrm{erg} \mathrm{cm}^{-2} \mathrm{~s}^{-1}$ and a poor spectrum consistent with various models characterized the ASCA observation (Turner et al. 1997). Preliminary results on a 2001 XMM-Newton observation were presented by Matt (2002b), clearly showing that the source was reflection-dominated. We present here full results on this XMM-Newton observation, together with a later one. The two spectra are fully compatible each other and are well fitted by the same model adopted for NGC 7674. The only emission line apparent in the data is the neutral $\mathrm{Fe} \mathrm{K} \alpha$ line: Table 3 and Fig. 2 show the best fit parameters and the spectra. No significant variability is found in fluxes or spectral shape between the two observations. Moreover, the XMM-Newton lightcurves of each observation are consistent with a constant source. 


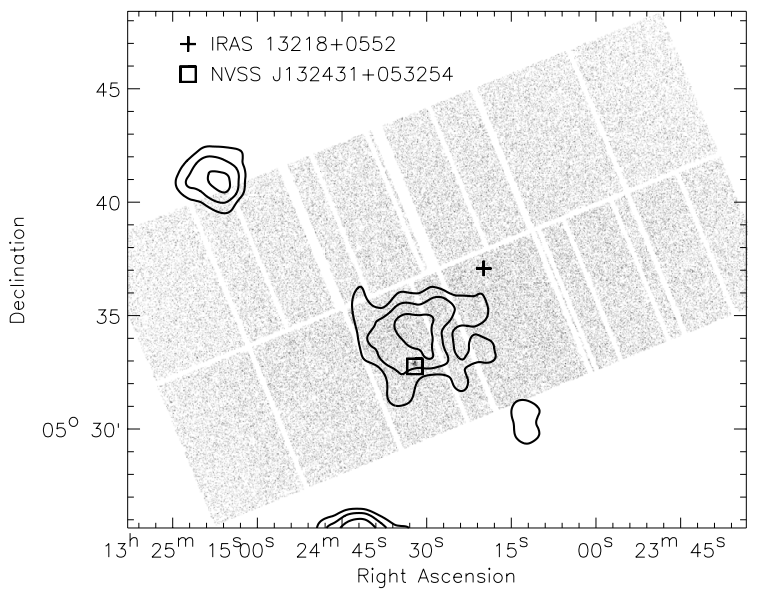

Fig. 3. IRAS 13218+0552: BeppoSAX contour levels superimposed over the EPIC pn field. The target source and a nearby object are marked with a cross and a square, respectively.

\subsection{IRAS $13218+0552$}

The source is the only object in our sample with a moderately large redshift and classified as a type 1. After the HEAO A-1 detection, the first X-ray observation of IRAS 13218+0552 was performed on July 2000 by BeppoSAX, which detected a source with a flux more than 60 times fainter and a low quality spectrum: $N_{\mathrm{H}}$ and $\Gamma$ are poorly constrained, but the spectrum seems to be steep $(\Gamma \sim 2)$. Interestingly enough, there seems to be an excess flux in the PDS, suggesting moderately Compton-thick absorption; however, at these count-rate levels $\left(0.08 \pm 0.05\right.$ counts $\left.\mathrm{s}^{-1}\right)$ confusion is a serious issue and we cannot draw any definite conclusion.

However, the XMM-Newton observation failed to detect IRAS 13218+0552, with an upper limit of $3.9 \times$ $10^{-14} \mathrm{erg} \mathrm{cm}^{-2} \mathrm{~s}^{-1}$ in the EPIC pn. The closest bright source, located at $\simeq 5$ arcmin from the target, corresponds to the radio source NVSS J132431+053254 (see Table 6). The X-ray spectrum of this source is fully consistent with that of the source observed by BeppoSAX (an unabsorbed powerlaw with $\Gamma \simeq 2$ ), even if its flux is significantly lower, being $5.3 \times 10^{-14} \mathrm{erg} \mathrm{cm} \mathrm{cm}^{-2} \mathrm{~s}^{-1}$. We show in Fig. 3 the contour plot of the MECS observation superimposed on the EPIC pn image, marking the positions of IRAS $13218+0552$ and NVSS J132431+053254: it seems likely that the source observed by BeppoSAX is the latter, so that the target object was undetected also in this observation. Moreover, in a ROSAT observation of the same field, no source is apparent at the coordinates of IRAS $13218+0552$.

\subsection{NGC 1667}

After the X-ray detection by HEAO A-1 at a flux of $1.9 \times$ $10^{-11} \mathrm{erg} \mathrm{cm}^{-2} \mathrm{~s}^{-1}$, the source was not detected by GINGA and presented a very low flux in the ASCA observation (Turner et al. 1997; Pappa et al. 2001). Indeed, our re-analysis of the ASCA data shows that the source is practically undetected above $2 \mathrm{keV}$. Moreover, only an upper limit was found by the PSPC within the RASS (Rush et al. 1996).
Table 4. NGC 1667: best fit parameters for the observation analyzed in this paper (see text for details).

\begin{tabular}{c|c}
\hline \hline & XMM \\
\hline$F_{2-10}\left(10^{-12} \mathrm{erg} \mathrm{cm}^{-2} \mathrm{~s}^{-1}\right)$ & $0.10 \pm 0.03$ \\
$\Gamma$ & $1.7^{*}$ \\
$\Gamma_{\mathrm{s}}$ & $3.2_{-0.3}^{+0.4}$ \\
$E W_{6.4}(\mathrm{eV})$ & $<600$ \\
$\chi^{2} /$ d.o.f. & $17 / 14$ \\
\hline
\end{tabular}

${ }^{*}$ Fixed.

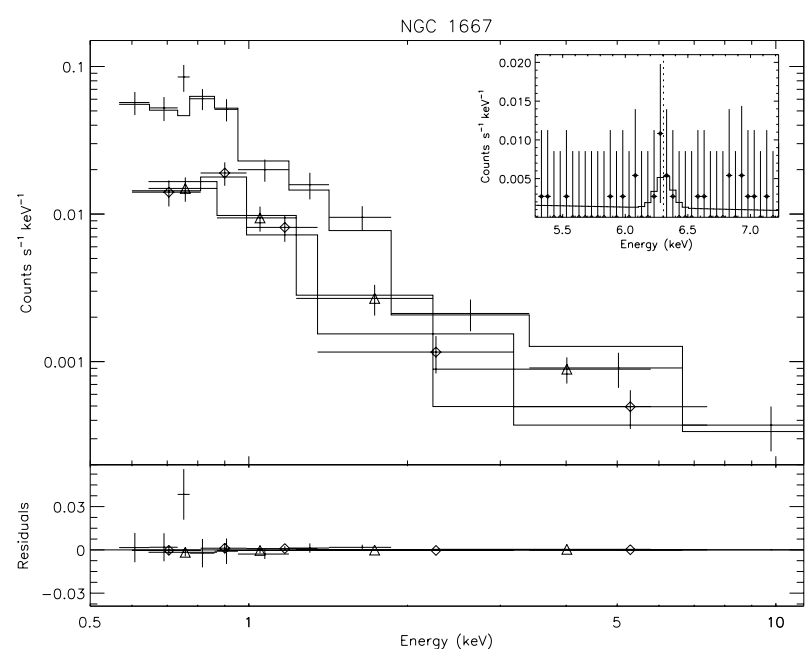

Fig. 4. NGC 1667: XMM-Newton spectra (pn: crosses, MOS1: diamonds, MOS2: triangles) and best fit model. In the inset: "local fit" on the background-subtracted, unbinned pn spectrum around the restframe iron line energy, marked with a broken line (see text for details).

A low flux level similar to the one found by ASCA characterizes the XMM-Newton observation. A fit with a simple powerlaw leads to a very steep photon index $(\Gamma \simeq 3$ ), but with large residuals on the softer and the harder parts of the spectrum, resulting in an unacceptable reduced $\chi^{2}$, greater than 3 . On the other hand, the large ratio between the [OIII] and the far infrared (IR) fluxes, together with a relatively low X-ray flux, hint to a Compton-thick source (see Tables 1, 5 and Sect. 4.2 for details). Therefore, we tried a model with a pure reflection component and a soft excess: now the fit is perfectly acceptable $\left(\chi^{2}=17 / 14\right.$ d.o.f.: see Table 4 and Fig. 4$)$. The model includes an emission line at $0.87_{-0.02}^{+0.03} \mathrm{keV}$ (likely Ne IX K $\alpha$ ), required at the $97 \%$ confidence level according to F-test. The presence of a strong iron line is suggested by the "local fit" (see inset in Fig. 4), with a flux of $1.0_{-0.6}^{+1.4} \times 10^{-6} \mathrm{ph} \mathrm{s}^{-1}$, consistent with the upper limit found in the global fit. This would correspond to an $E W$ of $\simeq 600 \mathrm{eV}$. The observed $2-10 \mathrm{keV}$ flux is $1 \times 10^{-13} \mathrm{erg} \mathrm{cm}^{-2} \mathrm{~s}^{-1}$ and no significant short-term variability trends are present in the lightcurve.

\section{Discussion}

\subsection{Classification of the objects in the sample}

All the sources included in our sample turn out to be likely Compton-thick. While the spectral analysis presented in this paper strongly favour this scenario at least for NGC 7674 and 

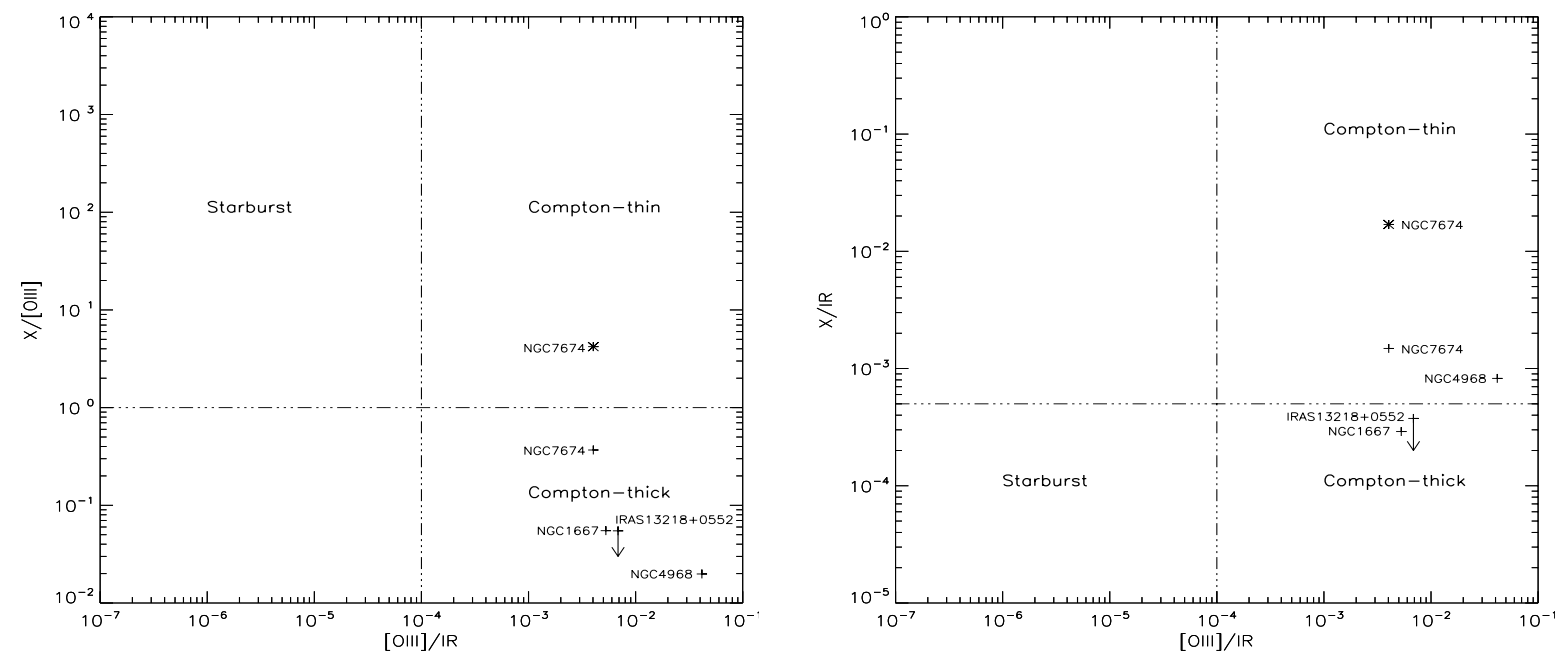

Fig. 5. $F_{\mathrm{X}} / F_{\mathrm{IR}}, F_{[\mathrm{OIII}]} / F_{\mathrm{IR}}$ and $F_{\mathrm{X}} / F_{[\mathrm{OIII}]}$ diagrams after Panessa \& Bassani (2002). See Tables 1 and 5 for data (the X-ray fluxes are taken from the XMM-Newton observations, except for NGC 7674, whose GINGA flux is also used and the relative point is marked by a star instead of a cross).

NGC 4968 (see Sect. 3), another test can be done on the basis of their [OIII], infrared (IR) and X-ray fluxes, as explained, for example, by Panessa \& Bassani (2002). Looking at the diagrams shown in Fig. 5, it is clear that all the four sources populate or are very close to the regions of the Compton-thick Seyfert galaxies. However, we note here that it should be better to define them reflection-dominated objects, since these diagrams cannot distinguish between highly obscured and "switchedoff" sources. Indeed, in at least one case, NGC 7674, the diagrams in Fig. 5 show that the source moved from the Compton-thin to the Compton-thick region between two X-ray observations, thus being a good "switched off" candidate. We will discuss this object further in Sect. 4.3.

\subsection{A re-assessment of LMA identifications}

None of the sources in our sample was caught in a high state, comparable to the one measured by HEAO A-1. Therefore, before speculating on large flux variations, we should first reassess the reliability of the LMA fluxes.

\subsubsection{Wrong identifications}

A first test is to check the optical identifications given by Grossan (1992) for the four sources in our sample. We reconstructed the rectangular HEAO A-1 error boxes using the coordinates reported by Wood et al. (1984) and overplotted the EPIC pn fields together with the position of the AGN which constitute our sample. The results are shown in Fig. 6. With the only exception of NGC 4968, all the sources fall largely outside of the 95\% LASS error box. Note that the actual error region is much smaller than the latter, because the LMA identifications also take into consideration the MC pointings. One of the sources, IRAS 13218+0552, was part of a 22-objects subsample of the LMA analyzed in detail by Remillard et al. (1993), who included a figure very similar to the one presented here. Nonetheless, he suggested that in this case (and in some others) the LASS error boxes should be considered as $\simeq 50 \%$ contours. However, it is clear that these identifications are much less robust than for other LMA sources.

If this is the case, we should also look for contamination by nearby sources, which could contribute to the total flux measured by HEAO A-1. The LASS error boxes shown in Fig. 6 are overplotted on the RASS images of the same fields. No bright source lies inside any of the HEAO A-1 boxes. Moreover, we measured fluxes of the brightest objects present in the EPIC fields of each target source. The results are shown in Table 6: none of these sources have X-ray fluxes comparable to the one measured by the LMA and are all (with the exception of NVSS J132431+053254 and SDSS J132442.44+052438.9 in the otherwise empty field of IRAS 13218+0552), much dimmer than the AGN. Even if some of them can be highly variable sources, we have no evidence that their fluxes could have significantly contaminated the LASS count rates.

\subsubsection{Wrong fluxes}

Even if the optical identification given by Grossan (1992) are very uncertain, there is no clear evidence of contamination from other sources. Therefore, it is possible that the LMA fluxes are wrong. Indeed, there are two reasons to suspect that. The first one is that the fluxes of these sources are among the lowest in the sample and, in the cases of IRAS 13218+0552 and NGC 1667, they are just above the flux limit of the sample itself, with errors around $20 \%$. A second issue refers to the fact that HEAO A-1, due to an hardware failure, lost all its spectral resolution, so that it should be considered as a "large X-ray photometer over a range of 1-20 keV", to use the words of Grossan (1992). The measured count rates were then transformed to a flux density at $5 \mathrm{keV}$ adopting an empirical flux conversion derived from a comparison with the HEAO A-2 instrument and assuming a spectral shape of a powerlaw with a photon index of 1.7. The last assumption gives reasonable results for unabsorbed or even moderately absorbed AGN, 

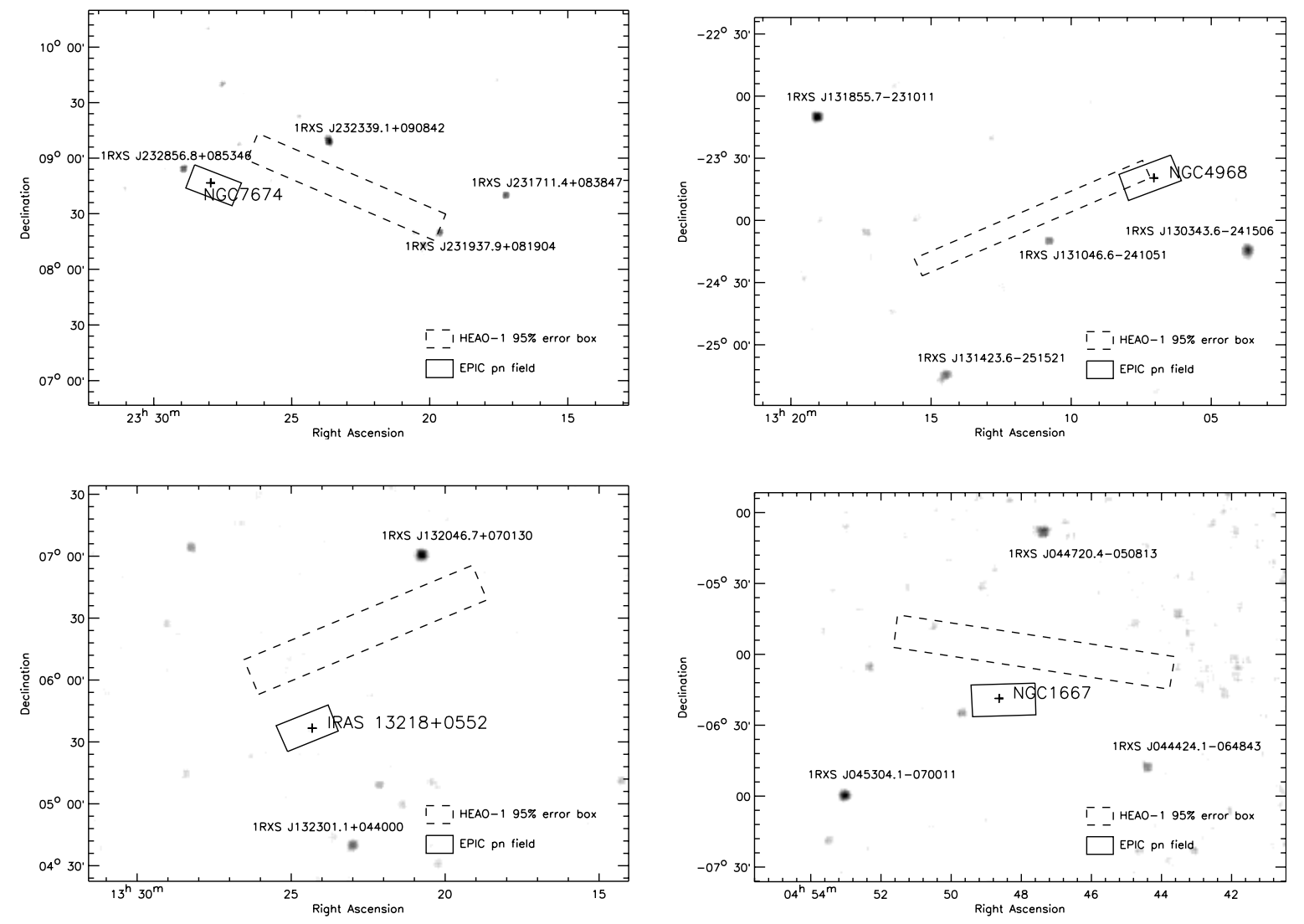

Fig. 6. HEAO A-1 95\% error boxes and EPIC pn fields of the XMM-Newton observations analyzed in this paper overplotted on the RASS images. A cross points to the coordinates of the LMA counterpart, while the brightest RASS sources are labelled (see text for details).

but can lead to completely wrong fluxes in Compton-thick sources. If the primary continuum is blocked up to $10 \mathrm{keV}$ or higher energies, the measured LASS count rates are totally unrelated to the $2-10 \mathrm{keV}$ flux of the source, because they refer, in an unknown way, to the total band of the instrument, up to $20 \mathrm{keV}$.

We performed a rough test with XSPEC to see if this scenario is consistent with the measured PDS fluxes in the two sources observed by BeppoSAX. We first calculated a 1-20 keV count-rate (arbitrarily normalized) which reproduced the 2-10 keV HEAO flux with a model consisting of an unabsorbed powerlaw with $\Gamma=1.7$. Then, we produced a model consisting of a bare Compton-reflection component (with a 2-10 keV flux like the one observed by XMM-Newton) and a highly obscured $\left(N_{\mathrm{H}}\right.$ of several $\left.10^{24} \mathrm{~cm}^{-2}\right)$ powerlaw, yielding the same count-rate. The resulting 10-20 keV flux is, for both NGC 7674 and IRAS 13218+0552, a factor $\simeq 100$ larger than the one observed by BeppoSAX, showing that, at least in these objects, it is unlikely that the count-rate observed by HEAO A-1 can be interpreted in terms of high energy emission of an otherwise obscured object. We note that our test assumes a flat response of the detector in the whole band $1-20 \mathrm{keV}$. In a more realistic case, where the instrumental effective area decreases at high energies, the difference between the expected flux and the one observed by the PDS would be larger.

In conclusion, even if we do not find any conclusive evidence against the identification or the flux of the sources in our sample, the above-mentioned issues may affect, in principle, all the low-flux objects in the Grossan (1992) catalog, which should be treated with due caution. However, it is worthwhile stressing that the criterion adopted in our sample clearly puts a strong bias towards potentially fake HEAO A-1 identifications or detections, since all the targets were not confirmed at the same flux level by other observations. For our purposes, all the preceding discussion does not allow us to speculate on large X-ray variations of the sources in our sample on the basis of their HEAO A-1 fluxes only. In particular, NGC 4968 is the only object which lies reasonably close to the LMA error box, but, apart from its LMA flux, it does not present any significant variation for several years, similarly to NGC 1667 (see Table 5). On the other hand, if we relax the constraints given by the HEAO error boxes, as suggested by Remillard et al. (1993), in one case, NGC 7674, the LMA flux could be in principle suggestive of a past high flux state of the source, considering the following GINGA flux, which is significantly larger than the BeppoSAX and the XMM-Newton ones. Finally, another object, IRAS $13218+0552$, is not detected at all by XMM-Newton. These two sources deserve some further comments.

\subsection{NGC 7674: a switched-off source?}

Even not taking into account the HEAO A-1 observation, the source underwent a flux loss by a factor $\gtrsim 10$ in the 7 years 
Table 5. The X-ray history of the four sources in our sample.

\begin{tabular}{|c|c|c|c|c|c|}
\hline & $\begin{array}{l}\text { HEAO A-1 } \\
\text { Flux [Date] }\end{array}$ & $\begin{array}{c}\text { GINGA } \\
\text { Flux [Date] }\end{array}$ & $\begin{array}{c}\text { ASCA } \\
\text { Flux [Date] }\end{array}$ & $\begin{array}{l}\text { BeppoSAX } \\
\text { Flux [Date] }\end{array}$ & $\begin{array}{l}\text { XMM-Newton } \\
\text { Flux [Date] }\end{array}$ \\
\hline NGC 7674 & $24 \pm 5[1977 / 78]^{a}$ & $8 \pm 2[1989]^{b}$ & - & $0.75 \pm 0.06[1996]^{d}$ & $0.70 \pm 0.07[2004]^{d}$ \\
\hline NGC 4968 & $22 \pm 3[1977 / 78]^{a}$ & $<5[1989]^{b}$ & $0.2_{-0.1}^{+0.4}[1994]^{d}$ & 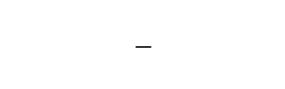 & $\begin{array}{l}0.27 \pm 0.08[2001]^{d} \\
0.23 \pm 0.08[2004]^{d}\end{array}$ \\
\hline IRAS $13218+0552$ & $18 \pm 5[1977 / 78]^{a}$ & - & - & $0.28 \pm 0.06^{*}[2000]^{d}$ & $<0.039[2004]^{d}$ \\
\hline NGC 1667 & $19 \pm 4[1977 / 78]^{a}$ & $\begin{array}{l}<7[1988]^{c} \\
<3[1990]^{c}\end{array}$ & $<0.15[1994]^{d}$ & - & $0.10 \pm 0.03[2004]^{d}$ \\
\hline
\end{tabular}

Fluxes are in $10^{-12} \mathrm{erg} \mathrm{cm}^{-2} \mathrm{~s}^{-1}(2-10 \mathrm{keV}) .{ }^{*}$ This flux is likely that of a nearby source, NVSS J132431+053254 (see text for details). References: ${ }^{a}$ Based on the HEAO A-1 count rates and conversion factor given in Wood et al. (1984); ${ }^{b}$ Awaki et al. (1991); ${ }^{c}$ Polletta et al. (1996) and references therein, but see also Sect. 2.2.1; ${ }^{d}$ This paper.

Table 6. Main data on the brightest sources in the EPIC fields analysed in this paper (see text for details).

\begin{tabular}{|c|c|c|c|c|c|}
\hline Source & Identification $^{a}$ & RA Dec (J2000) & Distance $^{b}$ & $F_{0.5-2 \mathrm{keV}^{c}}$ & $F_{2-10 \mathrm{keV}^{c}}$ \\
\hline 7674-X1 & XMMU J232757.4+084744 ${ }^{1}$ & $23^{\mathrm{h}} 27^{\mathrm{m}} 57^{\mathrm{s}} .4+08^{\circ} 47^{\prime} 44^{\prime \prime}$ & 1.1 & 0.62 & 0.22 \\
\hline $7674-X 2$ & 1WGA J2328.1+0849 2 & $23^{\mathrm{h}} 28^{\mathrm{m}} 07^{\lessgtr} 3+08^{\circ} 49^{\prime} 07^{\prime \prime}$ & 3.6 & 0.56 & 1.4 \\
\hline $7674-X 3$ & XMMU J232728.0+085206 ${ }^{1}$ & $23^{\mathrm{h}} 27^{\mathrm{m}} 28^{\mathrm{s}} .0+08^{\circ} 52^{\prime} 06^{\prime \prime}$ & 7.1 & 0.71 & 0.28 \\
\hline $7674-\mathrm{X} 4$ & 1WGA J2327.4+0845 2 & $23^{\mathrm{h}} 27^{\mathrm{m}} 26^{\mathrm{s}} .7+08^{\circ} 45^{\prime} 11^{\prime \prime}$ & 7.6 & 1.4 & 0.035 \\
\hline $7674-X 5$ & 2 MASX J23282907+0853013 3 & $23^{\mathrm{h}} 28^{\mathrm{m}} 28^{\mathrm{s}} .9+08^{\circ} 52^{\prime} 58^{\prime \prime}$ & 10.2 & 1.6 & 1.4 \\
\hline IRAS-X1 & NVSS J132431+053254 ${ }^{4}$ & $13^{\mathrm{h}} 24^{\mathrm{m}} 32^{\mathrm{s}} .0+05^{\circ} 32^{\prime} 51^{\prime \prime}$ & 5.2 & 0.48 & 0.53 \\
\hline IRAS-X2 & SDSS J132442.44+052438.9 ${ }^{5}$ & $13^{\mathrm{h}} 24^{\mathrm{m}} 42^{\mathrm{s}} .7+05^{\circ} 24^{\prime} 37^{\prime \prime}$ & 13.7 & 4.8 & 12.2 \\
\hline $1667-X 1$ & XMMU J044835.7-061810 ${ }^{1}$ & $04^{\mathrm{h}} 48^{\mathrm{m}} 35^{\mathrm{s}} .7-06^{\circ} 18^{\prime} 11^{\prime \prime}$ & 0.95 & 0.21 & 0.11 \\
\hline $1667-\mathrm{X} 2$ & XMMU J044833.1-061716 ${ }^{1}$ & $04^{\mathrm{h}} 48^{\mathrm{m}} 33^{\mathrm{s}} .2-06^{\circ} 17^{\prime} 16^{\prime \prime}$ & 2.1 & 0.23 & 0.45 \\
\hline $1667-X 3$ & XMMU J044851.4-062135 ${ }^{1}$ & $04^{\mathrm{h}} 48^{\mathrm{m}} 51^{\mathrm{s}} .4-06^{\circ} 21^{\prime} 35^{\prime \prime}$ & 4.4 & 0.13 & 0.18 \\
\hline
\end{tabular}

${ }^{a}$ References: (1) This paper, (2) White et al. (2000), (3) Cutri et al. (2003), (4) Condon et al. (1998), (5) Strateva et al. (2003), ${ }^{b}$ Distance (in arcmin) of the source from the target, ${ }^{c}$ In units of $10^{-13} \mathrm{erg} \mathrm{cm}^{-2} \mathrm{~s}^{-1}$.

separating the GINGA and BeppoSAX observation, then remained fairly stable after other 8 years, when the XMMNewton observation was performed (see Table 5). Therefore, NGC 7674 may well represent a good candidate for being a switched-off object.

This is also supported by a spectral transition between the two flux states. Indeed, the GINGA spectrum was a featureless powerlaw, with only an upper limit to the $E W$ of the iron line $(80 \mathrm{eV})$ and an unconstrained value for the neutral absorbing column density. On the other hand, the BeppoSAX and XMM-Newton spectral analysis clearly show that the source is actually reprocessing-dominated, with a bare Compton reflection continuum at high energies, an iron line with a $E W \simeq$ $400 \mathrm{eV}$ and a soft excess at lower energies (see Fig. 7). Moreover, the source lies in the Compton-thick region in the diagnostic diagrams based on the X-ray, IR and [OIII] fluxes (see Fig. 5).

Such a transition between a transmission- to a reprocessingdominated spectral state would make NGC 7674 the sixth Seyfert 2 galaxy to show this behaviour (see Guainazzi et al. 2005a, and references therein). The time elapsed between the GINGA, BeppoSAX observation and XMM-Newton observations allows us to make an estimate of the distance of the

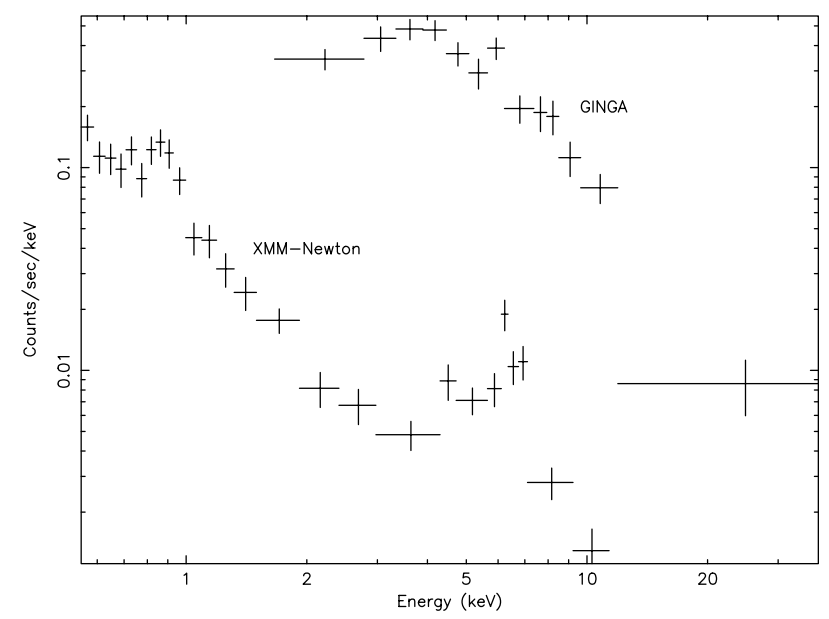

Fig. 7. NGC 7674: a comparison between the GINGA (upper) and XMM-Newton (lower) spectra, clearly showing the transition between a transition- to a reprocessing-dominated state (see text for details).

reflector from the Black Hole $(\mathrm{BH})$. If the primary source switched off shortly after the GINGA observation, the detection of a reflection echo 15 years after implies a distance of around 5 pc of the material responsible for it. On the other hand, if, 
instead, it switched off a little before the BeppoSAX observation, the same flux level measured 8 years later puts a lower limit of almost 3 pc. Since it is impossible to know if in these time intervals the source actually switched on again, care should be taken when considering these lower limits.

Another interesting piece of information comes from the ratio between the normalizations of the primary powerlaw in the Compton-thin state and the Compton reflection component in the reprocessing-dominated state, which leads to an estimate of the covering factor of the reflecting matter. In the case of NGC 7674, this value is approximately $R=1.5$, i.e. the subtended solid angle is around $3 \pi$. We have calculated the same ratio for all the "changing-look" Seyfert 2 sources in the literature and found values ranging from 0.8 to 1.8 (except for the extreme case of NGC 6300 , but in this case the primary continuum is very variable and it is difficult to calculate $R$, Guainazzi 2002). It should be noted that these values must be considered as estimates of the true covering factor, because they are calculated assuming transmission and reflection component fluxes which were not measured simultaneously, thus not necessarily directly linked one to the other. Since in "changing-look" sources the Compton-thick material clearly does not intercept the line of sight, these values of $R$ should be compared to the ones found in Seyfert 1s, which are in the range $\simeq 0.5-1$ (see e.g. Perola et al. 2002; Bianchi et al. 2004): this is suggestive that the same material (likely the torus envisaged in Unification Models, Antonucci 1993) is responsible for the reflection components observed in the two classes of objects.

\subsection{A misclassified Compton-thick object: IRAS 13218+0552}

While the HEAO A-1 flux is likely the result of a misidentification and the BeppoSAX flux also probably belongs to a nearby source (see previous section), the non-detection of IRAS $13218+0552$ by XMM-Newton still makes this source peculiar. Indeed, the upper limit derived for its X-ray flux, together with the IR and [OIII] fluxes, clearly puts this object among the Compton-thick sources (see Fig. 5). This is at odds with its current optical classification. Indeed, the presence of emission lines with components broader than $5000 \mathrm{~km} \mathrm{~s}^{-1}$ and the stellar-like appearance induced Low et al. (1988) to classify the source as a QSO. However, it was soon clear that IRAS $13218+0552$ was peculiar and its extremely red IR continuum won it the name of "reddest known quasar" (Low et al. 1989). Moreover, Remillard et al. (1993) reported anomalously broad [OIII] emission lines $\left(F W H M \simeq 3500 \mathrm{~km} \mathrm{~s}^{-1}\right)$, likely the result of some substructure in their profile. An asymmetric $[\mathrm{OIII}]$ profile, due to a blueshifted component, was also reported by Zheng et al. (2002).

The HST image in the $R$ band is strongly suggestive of a merger between two galaxies in its latest stage, according to Boyce et al. (1996). They also noted that, given its low nuclear luminosity, it should be strictly classified as a Seyfert 1, instead of a QSO, but its colors clearly points toward a very large obscuration, so that it is very likely that it hosts a buried QSO. An extreme velocity outflow (EVOF) component was finally unambiguously found in the $\mathrm{H} \beta$ and [OIII] line profiles, with velocities of the order of $2000 \mathrm{~km} \mathrm{~s}^{-1}$ (Lípari et al. 2003). This detection, together with the previous results, led these authors to conclude that IRAS $13218+0552$ is likely the result of a recent merging process and the nuclear energy is due to the composite activity of a hidden QSO and a starburst, the latter implied also by the tentative detection of Wolf-Rayet features. Indeed, the starburst component could even be the dominant one, as suggested by the optical emission line ratios reported by Kim et al. (1998) ${ }^{3}$.

In conclusion, the original classification as a QSO/Seyfert 1 was probably the result of a misinterpretation of the EVOF component as emission from the Broad Line Region. Therefore, the source should be re-classified as a type 2 object, Compton-thick in the X-rays and likely surrounded by a massive starburst. Within this scenario, it is possible that the PDS excess detected in the BeppoSAX observation (whose MECS spectrum is instead likely dominated by a nearby source: see Sect. 3.3) refers to the nuclear emission of the buried AGN, which pierces through a very large gas column density. However, if an AGN is actively heating the circumnuclear dust, an infrared compact source should be seen with the high spatial resolution imaging provided by $H S T$ at $1.6-2.2 \mu \mathrm{m}$, as seen in similar Compton-thick nearby Seyfert galaxies such as NGC 1068 (Thompson et al. 2001).

\section{Conclusions}

We have selected a sample of 4 AGN, included in the Grossan (1992) catalog, which showed in subsequent observations a flux much lower than the one measured with HEAO A-1, thus being good candidates for being "changing-look" sources. None of the sources was caught in a high flux state during the XMM-Newton observations. We have shown that, for all the sources, potential problems with the HEAO A-1 source identification and flux measurement prevent us to be certain that the HEAO A-1 data represent a putative "high" state for these objects.

However, based on the high flux state of its GINGA observation, a factor of ten higher than in the BeppoSAX and XMM-Newton observation, NGC 7674 represents probably the sixth known case of a "changing-look" Seyfert 2 galaxy. This is also supported by a spectral transition between a transmissionto reprocessing-dominated state between the two observations. From the X-ray variability pattern, we can estimate a lower limit of a few parsec to the distance of the reflecting material.

Finally, one of the sources, IRAS 13218+0552, was not detected by XMM-Newton, despite being currently classified as a Seyfert 1 with a large [OIII] flux. However, the original classification was likely to be affected by an outflow component in the emission lines. The object likely harbors an highly obscured AGN and should be re-classified as a type 2 source.

\footnotetext{
3 The previous classification as a type 1 object prevented these authors to use, in the case of IRAS $13218+0552$, the diagnostic diagrams plotted in Fig. 2 of their paper. However, once the presence of broad lines is excluded, we are allowed to use these line ratios to discriminate between a starburst- or AGN-dominated object.
} 
Acknowledgements. We would like to thank the referee, S. Lumsden, for his valuable suggestions. This paper is based on observations obtained with XMM-Newton, an ESA science mission with instruments and contributions directly funded by ESA Member States and the USA (NASA).

\section{References}

Antonucci, R. 1993, ARA\&A, 31, 473

Awaki, H. 1991, Ph.D. Thesis

Awaki, H., \& Koyama, K. 1993, Adv. Space Res., 13, 221

Awaki, H., Koyama, K., Inoue, H., \& Halpern, J. P. 1991, PASJ, 43, 195

Bassani, L., Dadina, M., Maiolino, R., et al. 1999, ApJS, 121, 473

Bianchi, S., Matt, G., Balestra, I., Guainazzi, M., \& Perola, G. C. 2004, A\&A, 422, 65

Bianchi, S., Miniutti, G., Fabian, A. C., \& Iwasawa, K. 2005, MNRAS, 360, 380

Boyce, P. J., Disney, M. J., Blades, J. C., et al. 1996, ApJ, 473, 760

Brinkman, A. C., Kaastra, J. S., van der Meer, R. L. J., et al. 2002, A\&A, 396, 761

Butcher, J. A., Stewart, G. C., Warwick, R. S., et al. 1997, MNRAS, 291, 437

Cash, W. 1976, A\&A, 52, 307

Condon, J. J., Cotton, W. D., Greisen, E. W., et al. 1998, AJ, 115, 1693

Cutri, R. M., Skrutskie, M. F., van Dyk, S., et al. 2003, VizieR Online Data Catalog, 2246, 0

Gabriel, C., Denby, M., Fyfe, D. J., et al. 2004, in Astronomical Data Analysis Software and Systems (ADASS) XIII, ASP Conf. Ser., 314,759

Gilli, R., Maiolino, R., Marconi, A., et al. 2000, A\&A, 355, 485

Grossan, B. A. 1992, Ph.D. Thesis

Guainazzi, M. 2002, MNRAS, 329, L13

Guainazzi, M., Nicastro, F., Fiore, F., et al. 1998, MNRAS, 301, L1

Guainazzi, M., Matt, G., Fiore, F., \& Perola, G. C. 2002, A\&A, 388, 787

Guainazzi, M., Rodriguez-Pascual, P., Fabian, A. C., Iwasawa, K., \& Matt, G. 2004, MNRAS, 355, 297

Guainazzi, M., Fabian, A. C., Iwasawa, K., Matt, G., \& Fiore, F. 2005a, MNRAS, 356, 295

Guainazzi, M., Matt, G., Perola, G. C., et al. 2005b, A\&A submitted

Gursky, H., Bradt, H., Doxsey, R., et al. 1978, ApJ, 223, 973

Hayashida, K., Inoue, H., Koyama, K., Awaki, H., \& Takano, S. 1989, PASJ, 41, 373

Iwasawa, K., Maloney, P. R., \& Fabian, A. C. 2002, MNRAS, 336, L71

Kim, D.-C. \& Sanders, D. B. 1998, ApJS, 119, 41

Kim, D.-C., Veilleux, S., \& Sanders, D. B. 1998, ApJ, 508, 627

Kinkhabwala, A., Sako, M., Behar, E., et al. 2002, ApJ, 575, 732

Levenson, N. A., Weaver, K. A., \& Heckman, T. M. 2001, ApJS, 133, 269
Lípari, S., Terlevich, R., Díaz, R. J., et al. 2003, MNRAS, 340, 289

Low, F. J., Cutri, R. M., Huchra, J. P., \& Kleinmann, S. G. 1988, ApJ, 327, L41

Low, F. J., Cutri, R. M., Kleinmann, S. G., \& Huchra, J. P. 1989, ApJ, 340, L1

Magdziarz, P. \& Zdziarski, A. A. 1995, MNRAS, 273, 837

Malaguti, G., Palumbo, G. G. C., Cappi, M., et al. 1998, A\&A, 331, 519

Matt, G. 2000, A\&A, 355, L31

Matt, G. 2002a, Royal Society of London Philos. Trans. Series A, 360, 2045

Matt, G. 2002b, in ASP Conf. Ser., 3

Matt, G., Fiore, F., Perola, G. C., et al. 1996, MNRAS, 281, L69

Matt, G., Guainazzi, M., \& Maiolino, R. 2003, MNRAS, 342, 422

Miller, J. S. \& Goodrich, R. W. 1990, ApJ, 355, 456

Panessa, F. \& Bassani, L. 2002, A\&A, 394, 435

Pappa, A., Georgantopoulos, I., Stewart, G. C., \& Zezas, A. L. 2001, MNRAS, 326, 995

Perola, G. C., Matt, G., Cappi, M., et al. 2002, A\&A, 389, 802

Piccinotti, G., Mushotzky, R. F., Boldt, E. A., et al. 1982, ApJ, 253, 485

Piconcelli, E., Jimenez-Bailón, E., Guainazzi, M., et al. 2004, MNRAS, 351, 161

Polletta, M., Bassani, L., Malaguti, G., Palumbo, G. G. C., \& Caroli, E. 1996, ApJS, 106, 399

Remillard, R. A., Bradt, H. V. D., Brissenden, R. J. V., et al. 1993, AJ, 105, 2079

Risaliti, G., Elvis, M., Fabbiano, G., Baldi, A., \& Zezas, A. 2005, ApJ, 623, L93

Rush, B., Malkan, M. A., Fink, H. H., \& Voges, W. 1996, ApJ, 471, 190

Sako, M., Kahn, S. M., Paerels, F., \& Liedahl, D. A. 2000, ApJ, 543, L115

Sambruna, R. M., Netzer, H., Kaspi, S., et al. 2001, ApJ, 546, L13

Smith, D. A. \& Done, C. 1996, MNRAS, 280, 355

Strateva, I. V., Strauss, M. A., Hao, L., et al. 2003, AJ, 126, 1720

Strüder, L., Briel, U., Dennerl, K., et al. 2001, A\&A, 365, L18

Thompson, R. I., Chary, R., Corbin, M. R., \& Epps, H. 2001, ApJ, 558, L97

Turner, M. J. L., Abbey, A., Arnaud, M., et al. 2001, A\&A, 365, L27

Turner, T. J., George, I. M., Nandra, K., \& Mushotzky, R. F. 1997, ApJS, 113, 23

Uttley, P., McHardy, I. M., Papadakis, I. E., Guainazzi, M., \& Fruscione, A. 1999, MNRAS, 307, L6

White, N. E., Giommi, P., \& Angelini, L. 2000, VizieR Online Data Catalog, 9031, 0

Wood, K. S., Meekins, J. F., Yentis, D. J., et al. 1984, ApJS, 56, 507

Young, S., Hough, J. H., Efstathiou, A., et al. 1996, MNRAS, 281, 1206

Zheng, X. Z., Xia, X. Y., Mao, S., Wu, H., \& Deng, Z. G. 2002, AJ, 124,18 